\title{
A study on the relationship between changes in serum hs-CRP levels and Chinese ischemic stroke subclassification
}

\author{
Lihe Yuan ${ }^{1}$, Yueming Yang ${ }^{1}$, Jiling $\mathrm{He}^{* 2}$ \\ ${ }^{1}$ Department of Neurology, The Third Affiliated Hospital of Inner Mongolia Medical University, Baotou, Inner Mongolia, China \\ ${ }^{2}$ The First Affiliated Hospital of Baotou Medical College, Baotou, Inner Mongolia, China
}

Received: January 5, 2018

Accepted: February 11, $2018 \quad$ Online Published: March 10, 2018

DOI: $10.14725 /$ dcc.v5n1p1

URL: http://dx.doi.org/10.14725/dcc.v5n1p1

\begin{abstract}
Objective: To study the relationship between changes in serum high sensitivity C-reactive protein (hs-CRP) levels and Chinese ischemic stroke subclassification (CISS), and explore the action mechanism of hs-CRP in the pathogenetic process of ischemic stroke.

Methods: The serum hs-CRP level was measured in all subjects (including the healthy). As to 177 ischemic stroke patients, they were subclassified based on causes and pathogenesis to explore the relationship between the serum hs-CRP level and CISS.

Results: The serum hs-CRP level in the ischemic stroke group was significantly higher than that in the control group $(p<.05)$. The difference of the serum hs-CRP levels in different Chinese ischemic stroke subtypes was of statistical significance $(p<.05)$. Conclusions: The level of serum hs-CRP is closely associated with the incidence of the ischemic stroke, and the difference of the serum hs-CRP levels in different Chinese ischemic stroke subtypes is of statistical significance.
\end{abstract}

Key Words: High sensitivity C-reactive protein, Ischemic stroke, Chinese ischemic stroke subclassification

High sensitivity C-reactive protein (hs-CRP), a proinflammatory factor related to the occurrence and development of atherosclerosis, plays an important role in the pathological mechanism of cerebral stroke. Cerebral strokes resulted from different causes show different clinical manifestations with treatment methods and prognosis differed. Therefore, the study on cerebral stroke subtypes is increasingly valued. Recently, Gao S et al. ${ }^{[1]}$ have proposed that, Chinese ischemic stroke subclassification (CISS) is a new stroke subclassification based on causes and pathogenesis and is widely used in clinical practice. With the application of CISS standards, this study is intended to observe and analyze the relationship between the level of hs-CRP and CISS, in order to provide an evidence for the diagnosis and treatment of ischemic strokes with different causes and pathogenesis.

\section{Objects and methods}

\subsection{Objects}

Patients who were hospitalized in Department of Neurology in our hospital, with ischemic stroke happened within 48 hours, verified by brain CT and MRI, were chosen as research objects, in conformance with the diagnostic criteria

\footnotetext{
*Correspondence: Jiling He; E-mail: dcc65@ncspress.com; Address: The First Affiliated Hospital of Baotou Medical College, Baotou, Inner Mongolia, China.
} 
established by the $4^{\text {th }}$ Congress of Chinese Cerebrovascular Diseases. Exclusion criteria were as follows: patients with autoimmune diseases, malignant tumors, infections, a history of trauma operation and usage of inflammationinhibiting drugs, immunosuppressants or hormones in recent 3 months. Besides, 180 healthy subjects were selected into the control group, the differences of gender and age between the control group and the stroke group were of no statistical significance.

\subsection{Methods}

\subsubsection{Test items}

On the morning of the second day after admission, fasting blood was required of all patients to determine hs-CRP concentration. Meanwhile, all patients were required to receive some examinations and tests, such as routine blood test, blood biochemical items, blood coagulation function, brain $\mathrm{CT}$ and/or MRI, ECG, carotid artery ultrasound, cerebral artery by color Doppler ultrasound and UCG etc., some patients need receive CTA and DSA examinations. Subjects in the control group were given some routine analyses to acquire results of routine blood tests and blood biochemical items, as well as hs-CRP concentration.

\subsubsection{CISS subtypes}

On the basis of medical history, clinical manifestations and some auxiliary examinations (laboratory and imageological examinations), all patients were subclassified into following types according to CISS etiological diagnosis: large artery atherosclerosis (LAA), penetrating artery disease (PAD), cardioembolic stroke (CS), other etiology (OE) and undetermined etiology (UE). Whereas, in LAA etiological diagnosis, these patients can also be subdivided into following subtypes according to pathogenesis: artery-artery embolism, hypoperfusion/insufficient ability of clearing embolus, parent artery plaque or thrombus occluding penetrating artery and mixed.

\subsubsection{Clinical neurological impairment degree evalua- tion}

National Institutes of Health Stroke Scale (NIHSS) was applied to clinical neurological impairment degree evaluation performed to these patients on admission. Clinical neurological impairment degree can be rated as follows: NIHSS $<4$ (slight), NIHSS 4-15 (moderate) and NIHSS > 15 (severe).

\subsubsection{Statistical treatment}

All data were represented by mean \pm standard deviation, with SPSS 17.0 statistical software applied to statistical analysis. The comparison between groups was made by use of $t$-test, and analysis of variance was applied to the correlation analysis between samples. The difference $(p<.05)$ was of statistical significance.

\section{Results}

\subsection{The comparison of hs-CRP level between the ischemic stroke group and the control group}

The serum hs-CRP level in patients with ischemic stroke was higher than that in healthy subjects, and the difference was of statistical significance $(p<.05$, see Table 1$)$.

Table 1: The comparison of hs-CRP level between the ischemic stroke group and the control group $(\bar{x} \pm \mathrm{s})$

\begin{tabular}{lll}
\hline Group & n & hs-CRP level (mg/L) \\
\hline Control Group & 180 & $2.57 \pm 1.41$ \\
Stroke Group & 177 & $7.59 \pm 4.40$ \\
\hline
\end{tabular}

\subsection{The comparison of hs-CRP level among differ- ent CISS subtypes}

On the basis of stroke causes, hs-CRP levels in each subtypes of patients were all increased: the level in CS was higher than that in the other subtypes $(p<.05)$, followed by LAA, and hs-CRP level in OE subtype was the lowest. The level of hs-CRP in PAD, OE and UE was significantly lower than that in LAA subtype $(p<.05$, see Table 2$)$.

Table 2: The comparison of hs-CRP level among different CISS subtypes $(\bar{x} \pm \mathrm{s})$

\begin{tabular}{lll}
\hline CISS Subtypes & $\mathbf{n}$ & hs-CRP level $(\mathbf{m g} / \mathbf{L})$ \\
\hline LAA & 98 & $8.05 \pm 4.22$ \\
PAD & 46 & $6.62 \pm 3.03$ \\
CS & 14 & $11.10 \pm 3.93$ \\
OE & 8 & $4.39 \pm 2.93$ \\
UE & 11 & $5.39 \pm 4.28$ \\
\hline
\end{tabular}

\subsection{The comparison of hs-CRP level among differ- ent CISS subtypes}

Based on CISS pathogenesis subtypes, in LAA subtype, the level of hs-CRP in artery-artery embolism group was higher than that in the other 3 groups $(p<.05$, see Table 3$)$. 
Table 3: The comparison of hs-CRP level among different CISS pathogenesis subtypes $(\bar{x} \pm \mathrm{s})$

\begin{tabular}{lll}
\hline CISS Pathogenesis Subtypes & $\mathbf{n}$ & hs-CRP level (mg/L) \\
\hline Artery-artery embolism group & 48 & $10.21 \pm 4.04$ \\
Parent artery plaque or thrombus occluding penetrating artery group & 22 & $7.60 \pm 3.39$ \\
Hypoperfusion/insufficient ability of clearing embolus group & 18 & $5.57 \pm 2.70$ \\
Mixed group & 10 & $3.15 \pm 1.20$ \\
\hline
\end{tabular}

2.4 The comparison of hs-CRP level among patients with different degrees of disease

The level of hs-CRP in severe patients (rated by NIHSS) was significantly higher than that in slight and moderate patients $(p<.05$, see Table 4$)$, indicating that the level of hs-CRP was related to clinical neurological impairment degree.

Table 4: The comparison of hs-CRP level among different NIHSS rated groups $(\bar{x} \pm \mathrm{s})$

\begin{tabular}{lll}
\hline NIHSS Rated Group & $\mathbf{n}$ & hs-CRP level $(\mathbf{m g} / \mathbf{L})$ \\
\hline Slight Group & 88 & $5.43 \pm 2.69$ \\
Moderate Group & 65 & $8.76 \pm 4.13$ \\
Severe Group & 24 & $12.33 \pm 2.90$ \\
\hline
\end{tabular}

\section{Discussion}

As an aspecific acute phase reaction protein, hs-CRP is involved in the occurrence and development of atherosclerosis, which is one of pathological bases that lead to cerebral stroke. ${ }^{[2]}$ Scholars at home and abroad have studied and found that, there exists a disagreement on the conclusion of the relationship between hs-CRP level and cerebral stroke. It is probable that different causes and pathogenesis lead to different results, and stroke subclassification based on causes and pathogenesis is significant to clinical diagnosis and treatment. Therefore, CISS was applied to this study in order to observe the level of hs-CRP in different ischemic stroke subtypes.

This study showed that serum hs-CRP level in patients with ischemic stroke was higher than that in the control group. DiNapoli et al. ${ }^{[3]}$ also reported that, the level of serum CRP in ischemic stroke patients started to increase in the early stage of the disease, and it was considered that inflammatory responses in the ischemic local lesions and the whole body stimulated hepatic cells to generate hs-CRP after the occurrence of cerebral stroke and resulted in the increase in the level of hs-CRP. The level of serum hs-CRP in patients with CISS subtypes of stroke was higher than that in the control group, and the difference in hs-CRP level among each subtype of patients was statistically significant. The results are similar with those reported by Zhang WW. ${ }^{[4]}$ In this study, hs-CRP level was the highest in CS group, followed by LAA, PAD, UE and OE. The results are similar with those acquired by Claes et al. and Fulton et al. ${ }^{[5,6]}$ in the research that hs-CRP level was measured on the basis of cause subclassification. With the help of some analyses, CS subtype of patients probably suffered from cardiovascular diseases, with large lesions, severe impairment and significant inflammatory responses due to large artery embolism, leading to a significant increase in hs-CRP level. Patients in LAA group also suffered from large artery lesions, therefore, hs-CRP level was also high in this group. However, PAD patients suffered from intracranial arteriolae and deep perforating artery lesions caused by hypertension and diabetes, with small lesions, less impairment and moderate inflammatory responses, leading to a slight increase in hs-CRP level. The difference in the level of hs-CRP showed that the intensity of inflammatory responses generated by different cause subtypes was different from each other, which indicated that the inflammatory process represented by hs-CRP was closely related to atherosclerosis lesions, and the measurement of hs-CRP had certain reference value for ischemic stroke subclassification.

In LAA subtype, on the basis of CISS pathogenesis, hsCRP level was significantly higher in artery-artery embolism group than that in the other three groups. It is probable that, vascular endothelial damage, caused by unstable plaque rupture in blood vessels in artery-artery embolism group, further magnifies inflammatory effects to make hsCRP level increased significantly. However, hs-CRP and pathogenesis are rarely reported. It requires further research and verification since CISS subtype is a newly proposed theory.

Patients with different degrees of ischemic stroke were grouped and observed, and the results showed that, those patients showed different levels of hs-CRP. Higher NIHSS rate will result in severer disease and higher level of hs-CRP, indicating that the increase in hs-CRP level is closely associated with the severity of disease, which is similar with the point proposed by literatures. ${ }^{[7,8]}$ It is analyzed that larger volume of infarction will result in severer neurological impairment degree, symptoms and local or systemic inflammatory responses, and stimulate hepatic cells to generate more hs-CRP.

All in all, inflammatory responses play an important role in the occurrence and development of ischemic stroke. Effects of hs-CRP on different types of ischemic stroke may differ, and they are closely related to stroke subtypes and the degree of disease. It is significant to stroke subtype and 
the judgment on the severity of disease, which has clinically important guidance significance in the active diagnosis and treatment of cerebral stroke. However, this study may be affected by the integrity of auxiliary examinations, less cases in some individual subgroups and researchers' knowledge on subclassification. It needs to be further studied by

\section{References}

[1] Gao S, Wang YJ, Xu AD, et al. Chinese ischemic stroke subclassification. Front Neurol. 2011; 2(1): 1-5. https://doi.org/10. 3389/fneur.2011.00006

[2] Liu LQ, Zeng QX. Study on the relationship between serum hs-CRP in hypertension patients and carotid atherosclerosis. Journal of Baotou Medical College. 2012; 28(5): 46-48.

[3] DiNapoli M, DiGianfilippa G, Sollecito A, et al. C-reactive protein and outcome after first - ever ischemic stroke. Stroke. 2000; 31(1): 238-239.

[4] Zhang WW, Zhou XY, Huang YH, et al. The changes and clinical significance of serum C-reactive protein in patients with different types of acute cerebral infarction. Chinese Journal of Laboratory Medicine. 2004; 27(11): 781-783. completing auxiliary examinations, increasing the number of samples and narrowing the difference in subclassification.

\section{Conflicts of Interest Disclosure}

The authors have no conflicts of interest related to this article.

[5] Claes L, Katarina J, Christian BD, et al. Serum C-reactive protein concentration and genotype in relation to ischemic stroke subtype. Stroke. 2006; 37: 2018-2023. PMid: 16809555. https://doi. org/10.1161/01.STR.0000231872.86071.68

[6] Fulton A, Stanton A, Moroney JT. C-reactive protein and carotid intimamedia thickness further define ischemic stroke subtype. Stroke. 2006; 37(2): 733 .

[7] Ladenvall C, Jood K, Blomstrand C, et al. Serum C-reactive protein concentration and genotype in relation to ischemic stroke subtype. Stroke. 2006; 37(8): 2018-2023. PMid: 16809555. https : //doi.org/10.1161/01.STR.0000231872.86071.68

[8] Luo Y, Wang Z, Li J. Serum CRP concentrations and severity of ischemic stroke subtype. Can J Neurol Sci. 2012; 39(1): 69-73. PMid: 22384498. https://doi.org/10.1017/S0317167100012713 